\title{
Absceso de Brodie, una patología de difícil diagnóstico
}

\author{
Brodie's abscess, a pathology difficult to diagnose
}

\author{
Isadora Silva C. ${ }^{a}$, María Jesús Figueroa G. ${ }^{b}$, Ismael Cañete C. ${ }^{b}$, Felipe Hodgson O. ${ }^{b}$, Alejandro Gündel P. ${ }^{b}$
}

aEscuela de Medicina. Facultad de Medicina, Pontificia Universidad Católica de Chile. Santiago, Chile

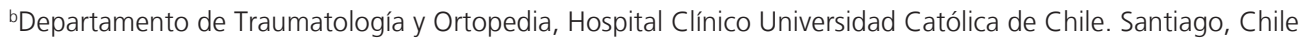

Recibido: 10 de febrero de 2020; Aceptado: 1 de agosto de 2020

¿Qué se sabe del tema que trata este estudio?

El absceso de Brodie es una osteomielitis subaguda de baja incidencia y de difícil diagnóstico, por lo que es imprescindible la sospecha clínica. Puede imitar varias enfermedades, ya que presenta una clínica sospechosa de tumores óseos, tanto benignos como malignos.

\section{¿Qué aporta este estudio a lo ya conocido?}

Se presenta un caso clínico, se describen las características epidemiológicas y etiológicas con la dificultad de su diagnóstico. Además, se realiza un análisis de la literatura de la patología.

\section{Resumen}

Las infecciones osteoarticulares agudas pediátricas constituyen patologías poco frecuentes, siendo de suma importancia realizar un diagnóstico precoz y tratamiento adecuado para evitar las complicaciones agudas o a largo plazo. El absceso de Brodie $(\mathrm{AB})$ es una forma de osteomielitis subaguda de baja incidencia y de difícil diagnóstico, por lo que es imprescindible la sospecha clínica. Objetivo: Presentar un caso de $\mathrm{AB}$ y describir las características etiológicas y clínicas de esta patología. Caso Clínico: Paciente 14 años, evaluado por dolor de muslo derecho de un mes de evolución, afebril y sin antecedente de trauma. Al examen físico no presentaba aumento de volumen, el rango de movimiento (ROM) de cadera derecha era doloroso y la sensibilidad estaba aumentada a la palpación superficial de banda iliotibial (BIT) derecha. Estudio radiológico sin alteraciones. Por persistir síntomas se solicitó ecografía de muslo que mostró irregularidad ósea cortical. Resonancia Magnética (RM) evidenció lesión en diáfisis de fémur derecho de probable origen tumoral o infeccioso. Parámetros inflamatorios sin alteraciones. Se realizó toma de biopsia y cultivos, aislando Staphylococcus aureus multisensible. Se procedió a aseo quirúrgico y terapia antibiótica endovenosa, evolucionando favorablemente. Conclusiones: La presentación clínica y laboratorio en $\mathrm{AB}$ pueden ser inespecíficas. El clínico no especialista debe tener un alto índice de sospecha de esta patología como posible diagnóstico diferencial en pacientes que persisten con dolor y presentan una alteración radiológica, incluso ante la ausencia de otros síntomas y parámetros inflamatorios normales. Es importante realizar una biopsia ósea para el diagnóstico diferencial de patologías tumorales.
Palabras clave:

Osteomielitis;

Absceso;

Brodie;

Infección

Correspondencia:

María Jesús Figueroa

mu iguer@uc.cl 


\begin{abstract}
Acute osteoarticular infections in children are rare pathologies, therefore early diagnosis and prompt treatment are crucial to avoid acute and long-term complications. Brodie's abscess (BA) is an uncommon type of subacute osteomyelitis, difficult to diagnose, so clinical suspicion is essential. Objective: To describe a case of Brodie's abscess and its etiological and clinical features. Clinical Case: A 14-year-old patient was seen at our clinic, who reported a one-month pain in the right thigh, with no history of fever or trauma. Physical examination revealed no volume increase, painful right hip range of motion, and increased sensitivity on superficial palpation of the right iliotibial band. X-rays where normal. Because of the pain persistence, an ultrasound was requested which showed a cortical irregularity. Magnetic resonance imaging (MRI) was performed and revealed a right femoral diaphysis, due to a possible bone tumor or an infectious process. Lab tests were normal. Biopsy and cultures were collected, identifying multi-sensitive Staphylococcus aureus. He was managed with debridement and intravenous antibiotics, responding positively. Conclusions: The BA's clinical features and lab tests are unspecific, therefore the non-specialist physician should strongly suspect this pathology as a possible differential diagnosis in patients who persist with pain and present imaging alterations, even when there are no other symptoms or normal inflammatory parameters. A bone biopsy is essential for the differential diagnosis of tumor pathologies.
\end{abstract}

\section{Keywords:}

Osteomyelitis;

Abscess;

Brodie;

Infection

\section{Introducción}

Las infecciones osteoarticulares agudas pediátricas tienen una baja frecuencia, $10-80$ por 100.000 niños $^{1}$, las cuales requieren un diagnóstico precoz y tratamiento adecuado para evitar las complicaciones agudas o morbilidad a largo plazo.

Existen 3 tipos de osteomielitis (OM): aguda, subaguda y crónica. Según su patogenia se pueden clasificar en hematógenas, inoculación directa y por contigüidad. La forma de presentación más común en niños es OM aguda, sin embargo, las formas subagudas hematógenas han ido en aumento, debido a una mayor resistencia del huésped, un descenso de la virulencia bacteriana y/o una exposición previa a antibióticos, lo que produce una lesión limitada al hueso ${ }^{2}$. El drenaje quirúrgico de estas infecciones óseas produce cultivos positivos en un $50-75 \%$ de los pacientes. $S$ aureus es el organismo causal más común (30-60\%), pero pueden encontrarse otros (Streptococcus, Pseudomonas, Haemophilus influenzae, Kingella kingae y Staphylococcus aureus coagulasa negativo $)^{2-4}$.

Por la siembra hematógena la infección afecta principalmente la metáfisis de los huesos largos (zona mejor irrigada y sin membrana basal), invade el hueso, inicia un proceso de inflamación que condiciona reabsorción ósea, se liberan enzimas proteolíticas, lo que va destruyendo el hueso y formando pus; esto aumenta la presión intramedular y el pus puede drenar al canal medular o al subperiostio. Debido a que en AB los gérmenes son de baja virulencia, este proceso es largo y reacciona formando hueso nuevo sobre el infectado, lo que se conoce como involucro. El hueso infectado si drena al periostio tiene interrupción de los vasos san- guíneos, por lo que se necrosa y forma un secuestro ${ }^{4-6}$.

Surgen por esto 2 formas clínicas en paciente pediátrico que dependen principalmente de la edad del paciente y la etiología. La forma infantil (6 meses a 4 años) corresponde al $85 \%$ de los pacientes con OM subagudas. Las técnicas diagnósticas mejoradas, como la reacción en cadena de la polimerasa (PCR), han demostrado Kingella kingae como principal microorganismo, con baja virulencia, con respuesta inflamatoria baja ${ }^{3,7,8}$. En la_forma juvenil ( $>4$ años) el causante principal es Staphylococcus aureus metilino sensible (SAMS). Es probable que exista una resistencia del huésped y que puede llegar a contener una infección ósea severa. La resistencia se puede explicar por la colonización frecuente de SAMS en niños, el 20\% de las personas están colonizadas permanentemente en la nariz ${ }^{46}$.

$\mathrm{El} \mathrm{AB}$ se ha descrito como una forma de OM subaguda de difícil diagnóstico debido a la ausencia de signos y síntomas inflamatorios sistémicos de la enfermedad aguda ${ }^{9-11}$. Tiene un inicio insidioso y se presenta principalmente con dolor ${ }^{3,7}$. El sitio primario de propagación bacteriana a menudo se desconoce, sin embargo, se planteó que la susceptibilidad ósea aumenta tras eventos traumáticos menores sin heridas abiertas o fracturas.

Esta forma de osteomielitis representa entre el 2,4\% y $42 \%$ de las infecciones óseas primarias en pacientes entre las edades de 6 meses y los 39 años, concentrándose principalmente en el rango de los 2-15 años ${ }^{4,7}$.

La incidencia se distribuye de manera desigual en el mundo, siendo los países desarrollados los más afectados. Las publicaciones son predominantemente de países desarrollados, lo que probablemente resulte en 
un sesgo de publicación sobre la incidencia mundial de la enfermedad ${ }^{10}$. En Chile no existe un registro sobre su incidencia.

Esta patología puede imitar varias enfermedades, ya que presenta signos y síntomas que harían sospechar tumores óseos, tanto benignos como malignos ${ }^{12,13}$.

En la literatura existen escasos estudios de buena calidad, siendo la mayoría reporte de casos. El objetivo es comunicar un caso de $\mathrm{AB}$, describir las características etiológicas y clínicas.

\section{Caso Clínico}

Paciente sexo masculino, 14 años con antecedentes de déficit atencional en tratamiento con Aripiprazol y Escitalopram, consultó por dolor en muslo derecho de un mes de evolución, afebril y sin trauma. Al examen físico no presentaba aumento de volumen, rango de movimiento (ROM) de cadera derecha doloroso, sensibilidad aumentada en la palpación superficial de banda iliotibial (BIT) derecha. Se realizó radiografía de pelvis AP-Löwenstein y fémur derecho AP-Lateral, las cuales no mostraron lesión ósea de fémur o alteraciones de cadera (Figura 1).

Se inició tratamiento con antiinflamatorios no esteroidales (AINES), reposo de actividad física, calor local, kinesioterapia y solicitud de ecografía de partes blandas.

Por persistir molestias, se realizó ecotomografía de partes blandas tres semanas después de la consulta inicial, la cual mostró una alteración cortical de la diáfisis femoral proximal, con área de pérdida de continuidad cortical y proliferación ósea irregular (Figura 2). Se complementó con radiografía de fémur, en donde se observó una lesión lítica mal delimitada, cortical, permeativa y de $8 \mathrm{~mm}$ en eje longitudinal (Figura 3 ).

Se consideró tumor óseo como diagnóstico diferencial, por lo que se decidió complementar con Resonancia Magnética (RM). En los hallazgos imagenológicos se observó una lesión intra cortical diafisaria proximal posterior de fémur derecho con caracteres altamente sugerentes de lesión estirpe inflamatorio-infeccioso (Absceso de Brodie intracortical), con extenso edema óseo medular reactivo, reacción periostal y edema-hiperemia de planos musculares vecinos (Figura 4).

Se realizó biopsia de tejido óseo con criterio oncológico por la existencia de una baja probabilidad de lesión neoplásica de células pequeñas o histiocitos $\mathrm{X}$, por la ubicación primariamente intracortical, además de la toma de cultivos.

Al momento del diagnóstico, los parámetros inflamatorios mostraron un recuento de leucocitos de $7.700 / \mu \mathrm{L}$, proteína $\mathrm{C}$ reactiva de $0,03 \mathrm{mg} / \mathrm{dL}$ y una velocidad de eritrosedimentación de $10 \mathrm{~mm} / \mathrm{hr}$., todos dentro de rangos normales.
A las 24 h se informó cultivo (+) de tejido óseo para Staphylococcus aureus e informe anatomopatológico reveló biopsia compatible con osteomielitis, sin evidencias de neoplasia.
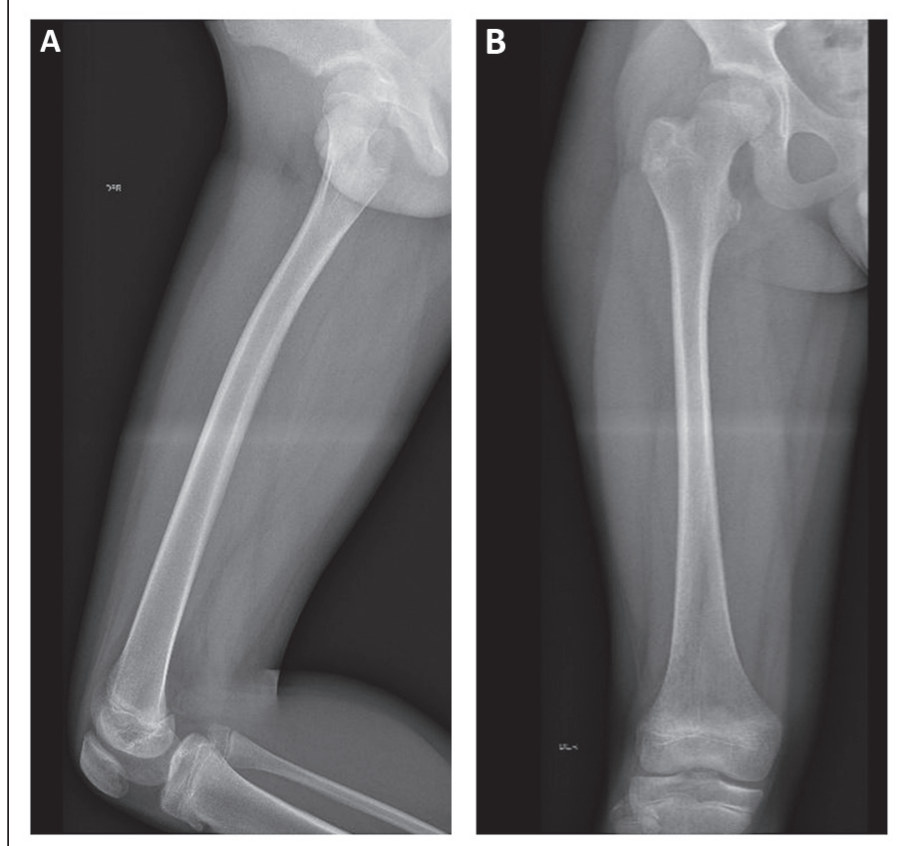

Figura 1. Rx de fémur derecho: A) lateral y B) AP. Sin evidencias de una lesión ósea traumática o destructiva.

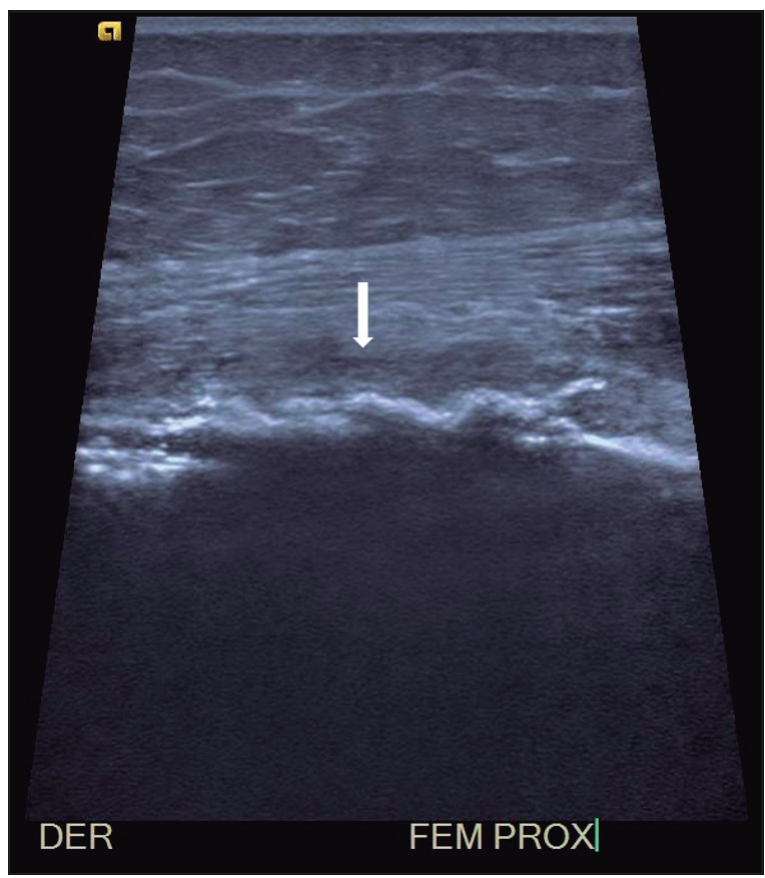

Figura 2. Ecotomografía de muslo derecho: se observa alteración e irregularidad de la cortical de fémur proximal derecho, asociado a reacción perióstica. 


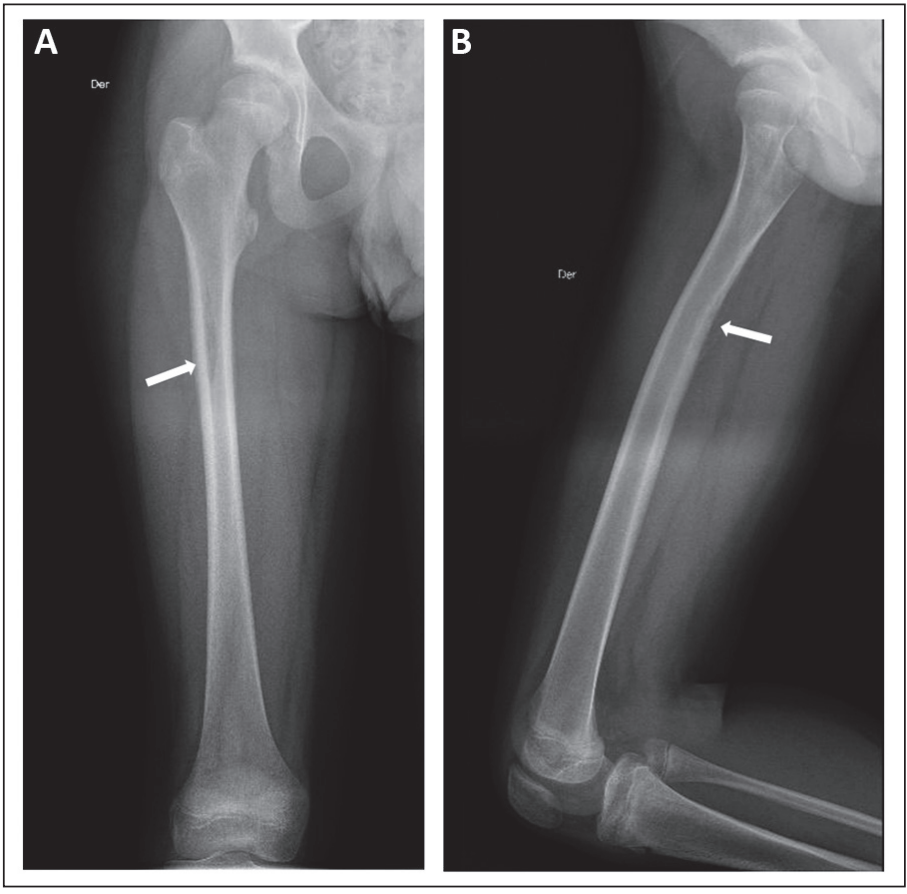

Figura 3. Rx de fémur: A) AP; B) Lateral. Se observa lesión lítica mal delimitada, cortical, permeativa, que mide aproximadamente $8 \mathrm{~mm}$ en el eje longitudinal y se acompaña de reacción perióstica inmadura.

Se realizó aseo quirúrgico presentando cultivos positivos para Staphylococcus aureus multisensible, indicándose tratamiento antibiótico endovenoso con Vancomicina y Ampicilina/sulbactam por 14 días, con buena respuesta clínica. Fue dado de alta hospitalaria y se indicó reposo relativo más antibióticos vía oral (Rifampicina $300 \mathrm{mg}$ cada $12 \mathrm{~h}$ y Ciprofloxacino $500 \mathrm{mg}$ cada $12 \mathrm{~h}$ ) durante 2 meses.

El paciente completó el tratamiento médico con antibióticos orales, obteniendo una mejoría clínica y radiográfica de la lesión. Siguió con controles médicos ambulatorios durante 1 año aproximadamente y se reintegró a la práctica deportiva.

\section{Discusión}

En la mayoría de los casos de AB los pacientes debutan con dolor persistente como principal síntoma, acompañado de sensibilidad local. Por lo general los síntomas están presentes durante 2 semanas o más, previo al diagnóstico. En el examen físico la sensibilidad localizada puede asociarse con calor, enrojecimiento o edema de los tejidos blandos con la afectación del hueso subcutáneo. El dolor puede ocurrir con el movimiento de la articulación adyacente, y puede haber derrame articular, pero estos suelen ser leves. Los músculos circundantes ocasionalmente muestran un compromiso inflamatorio ${ }^{14}$. El paciente de nuestro caso presentó dolor en muslo derecho durante un mes previo al momento de la consulta, ROM doloroso de cadera y sensibilidad aumentada a la palpación superficial de BIT, sin aumento de volumen.

Figura 4. RM de fémur derecho. A) Corte sagital STIR; B) Corte axial $\mathrm{T} 1$; C) Corte axial T1 con Gadolinio. En la cortical posterolateral del tercio proximal del fémur se observa una lesión intracortical (Flecha blanca) compatible con absceso de Brodie. Destaca el extenso edema óseo medular, periostal y músculos vecinos.

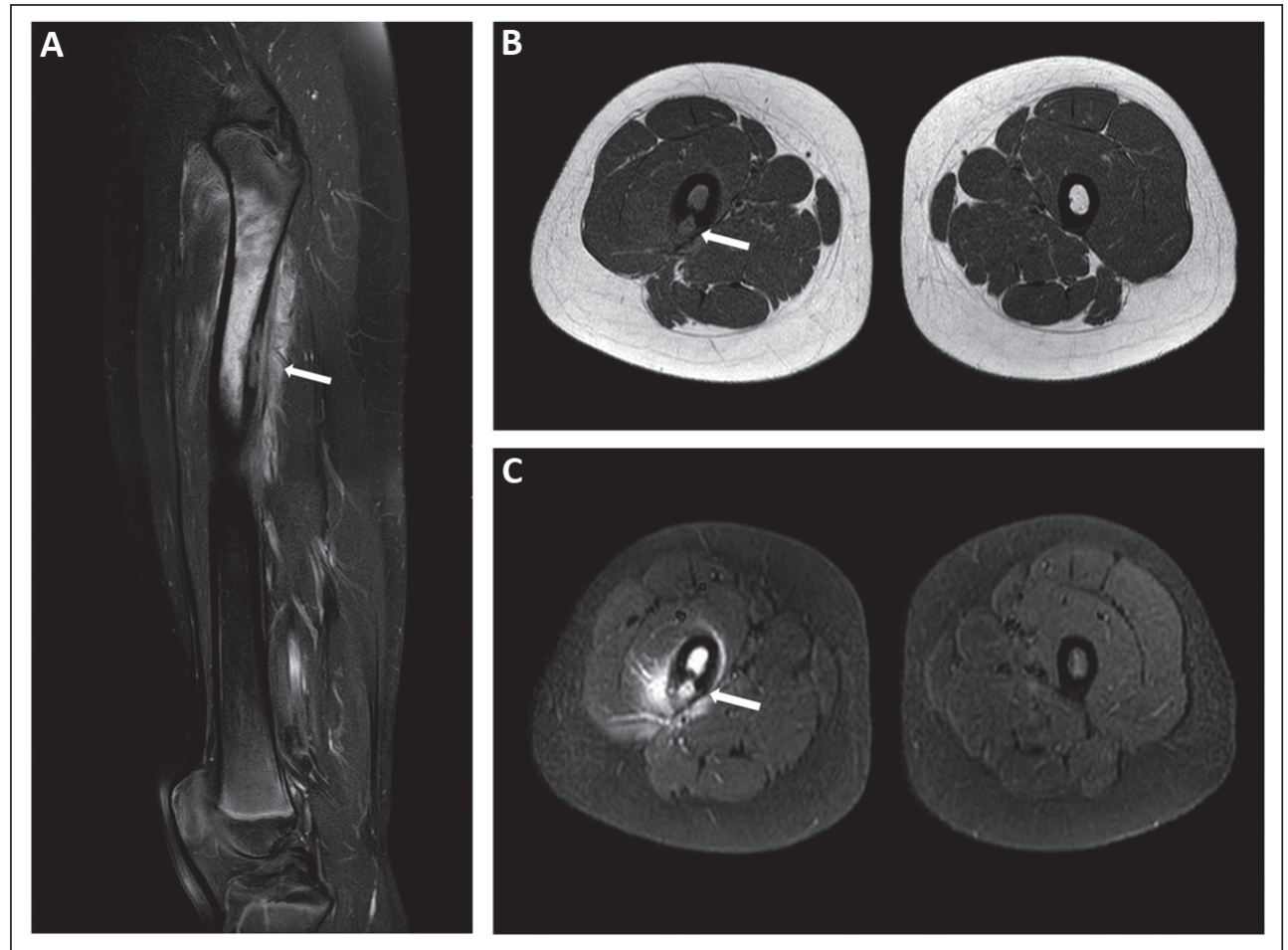


En los exámenes de laboratorio los hallazgos comunes suelen ser: hemocultivos negativos, recuento de leucocitos dentro del rango normal o ligeramente elevado y mediciones de la velocidad de sedimentación globular y proteína $\mathrm{C}$ reactiva ligeramente elevadas, pero pueden estar dentro de los rangos de referencia en el $30-50 \%$ de los pacientes ${ }^{2}$. En nuestro caso, los parámetros inflamatorios no presentaron alteraciones.

Se han descrito muchas técnicas de imagen para diagnosticar el absceso de Brodie; radiografías, tomografía computarizada, RM y medicina nuclear. La RM ha demostrado ser superior a la radiografía simple para diagnosticar osteomielitis en adultos, y en niños es la modalidad de elección para distinguir entre infección, tumor óseo y otras lesiones ${ }^{10,15}$. Sin embargo, esta técnica de imagen no se encuentra disponible en todos los hospitales, por lo que en ocasiones es necesario complementar el estudio radiológico con otros exámenes (ecografía, tomografía computarizada, cintigrama óseo). En nuestro caso, las radiografías iniciales no presentaron alteraciones. Al persistir el cuadro, se realizó una ecografía y nuevas radiografías que evidenciaban lesiones en la cortical diafisaria del fémur derecho. Se complementó el estudio con RM para distinguir un proceso infeccioso de un tumor óseo. En ella se observó un extenso edema óseo medular reactivo, reacción periostal y edema-hiperemia de planos musculares vecinos, sugerentes de un proceso infeccioso.

El AB clásico, descrito por primera vez por Sir Benjamin Brodie, corresponde a una lesión lítica metafisaria y esclerosis circundante bien delimitada. Actualmente la clasificación de Gledhill modificada por Roberts et al. ${ }^{16}$, permite la caracterización de los hallazgos radiológicos de las lesiones basado en su localización, morfología y similitud con neoplasias. En base a esta clasificación y las características radiográficas de neoplasias malignas o benignas, se pueden plantear posibles diagnósticos diferenciales en función de la edad del paciente, clínica y localización. Algunas neoplasias benignas serían el osteoma osteoide, granuloma eosinófilo y quiste óseo solitario. Mientras que el sarcoma de Ewing y metástasis son algunas de las neoplasias malignas $^{12,16}$

Según la clasificación de Roberts et al, las lesiones Tipo III y Tipo IV son diafisarias. Pueden presentar reacción perióstica con una sola capa o multilaminada, con o sin destrucción ósea. Además, se puede observar una inflamación de los tejidos blandos que recubren la lesión ${ }^{16}$. El caso clínico corresponde a una lesión tipo III cortical proximal de fémur con reacción perióstica.

En las imágenes de RM T1-W el signo de penumbra ayuda a excluir la presencia de un tumor y de esta manera, apoyar el diagnóstico de osteomielitis suba- guda. En ellas se observa una capa gruesa de tejido de granulación altamente vascularizado. Es una zona periférica discreta, con una intensidad de señal marginalmente mayor que la cavidad del absceso y del edema medular circundante, y de menor intensidad de señal que la médula ósea ${ }^{13,17}$.

El tratamiento de la OM hematógena subaguda es médico-quirúrgico. Teniendo en consideración el bajo rendimiento de los cultivos, la mayoría de las veces debemos iniciar un tratamiento ATB empírico, basado en la distribución de los gérmenes según edad y factores de riesgo. El tratamiento empírico debe incluir siempre cobertura de Staphylococcus aureus, si se logra identificar el agente, el tratamiento debe ajustarse según sensibilidad.

El tratamiento consiste fundamentalmente en un régimen secuencial de ATB endovenosos y luego orales. Actualmente la respuesta clínica y de laboratorio (niveles de proteína $\mathrm{C}$ reactiva), son usados para decidir el paso a ATB orales. La duración total del tratamiento no debe ser menor a las 6 semanas ${ }^{10}$.

El rol primario de la cirugía es drenar colecciones de material purulento, con lo cual se logra mejorar el ambiente local para permitir la llegada de ATB. Si el hueso no se regenera, puede generarse un defecto permanente. La cirugía también permite remover restos necróticos y así evita la evolución a la cronicidad. Básicamente consiste en incisión perióstica y remoción de todo el exudado y contenido necrótico y en defectos grandes se puede introducir perlas de cemento con antibiótico. Puede ser necesario repetir aseos quirúrgicos, según la evolución del paciente. Una vez controlada la infección algunos autores recomiendan la introducción de injerto óseo en el defecto ${ }^{18}$.

Realizando un adecuado tratamiento médico quirúrgico, se reportan tasas de éxito del 95 al 98\% de los $\operatorname{casos}^{10,18}$. En nuestro caso, se realizó aseo quirúrgico y tratamiento antibiótico endovenoso por 14 días, con buena respuesta clínica. Se continuó tratamiento oral antibiótico durante 2 meses, obteniendo una respuesta clínica exitosa.

\section{Conclusión}

La historia clínica, examen físico, parámetros inflamatorios y estudios de imágenes NO son suficientes para un diagnóstico de certeza del AB. El clínico no especialista debe tener un alto índice de sospecha de esta patología como posible diagnóstico diferencial en pacientes que persisten con dolor y presentan una alteración radiológica, incluso ante la ausencia de otros síntomas y parámetros inflamatorios normales. Es importante realizar una biopsia ósea para descartar patologías tumorales. 


\section{Responsabilidades Éticas}

Protección de personas y animales: Los autores declaran que los procedimientos seguidos se conformaron a las normas éticas del comité de experimentación humana responsable y de acuerdo con la Asociación Médica Mundial y la Declaración de Helsinki.

Confidencialidad de los datos: Los autores declaran que han seguido los protocolos de su centro de trabajo sobre la publicación de datos de pacientes.
Derecho a la privacidad y consentimiento informado: Los autores han obtenido el consentimiento informado de los pacientes y/o sujetos referidos en el artículo. Este documento obra en poder del autor de correspondencia.

\section{Conflicto de intereses}

Los autores declaran no tener conflicto de intereses.

\section{Referencias}

1. Arnold JC, Bradley JS. Osteoarticular Infections in Children. Infect Dis Clin North Am. 2015;29(3):557-74.

2. Jaramillo D, Dormans JP, Delgado J, Laor T, St Geme JW. Hematogenous osteomyelitis in infants and Children: Imaging of a Changing Disease. Radiology. 2017;283(3):629-43.

3. Hourston GJM, Kankam HKN, Mitchell PD, Latimer MD. Brodie abscess of the femoral capital epiphysis in a 2-year-old child caused by Kingella kingae. BMJ Case Rep. 2017;2017:3-5.

4. Blyth MJG, Kincaid R, Craigen MAC, Bennet GC. The changing epidemiology of acute and subacute haematogenous osteomyelitis in children. J Bone Jt Surg Ser B. 2001;83(1):99-102.

5. Spyropoulou V, Dhouib Chargui A, Merlini L, et al. Primary subacute hematogenous osteomyelitis in children: a clearer bacteriological etiology. J Child Orthop. 2016;10(3):241-6.

6. Shih HN, Shih LY, Wong YC. Diagnosis and treatment of subacute osteomyelitis. J Trauma. 2005;58(1):83-7.

7. Qi R, Colmegna I. Brodie abscess. CMAJ. 2017;189(3):E117.

8. Ruttan TK, Higginbotham E, Higginbotham N, Allen $\mathrm{CH}$, Hauger S. Invasive Kingella kingae resulting in a brodie abscess. J Pediatric Infect Dis Soc. 2015;4(2):e14-6.

9. Mehdinasab SA, Sarafan N, NajafzadehKhooei A. Primary subacute osteomyelitis of the greater trochanter. Arch Iran Med. 2007;10(1):104-6.

10. van der Naald N, Smeeing DPJ, Houwert RM, Hietbrink F, Govaert GAM, van der Velde D. Brodie's Abscess: A Systematic Review of Reported Cases. J Bone Jt Infect. 2019;4(1):33-9.

11. González-López JL, Soleto-Martín FJ, Cubillo-Martín A, et al. Subacute osteomyelitis in children. J Pediatr Orthop Part B. 2001;10(2):101-4.

12. Gledhill RB. Subacute osteomyelitis in children. Clin Orthop Relat Res. 1973;No. 96:57-69. Disponible en: http://www.ncbi. nlm.nih.gov/pubmed/4749831.

13. Shimose S, Sugita T, Kubo T, Matsuo T,
Nobuto H, Ochi M. Differential diagnosis between osteomyelitis and bone tumors. Acta radiol. 2008;49(8):928-33.

14. Stephens MM, MacAuley P. Brodie's abscess. A long-term review. Clin Orthop Relat Res. 1988;(234):211-6.

15. Agrawal P, Sobti A. A Brodie's Abscess of Femoral Neck Mimicking Osteoid Osteoma: Diagnostic Approach and Management Strategy. Ethiop J Health Sci. 2016;26(1):81-4.

16. Roberts JM, Drummond DS, Breed AL, Chesney J. Subacute hematogenous osteomyelitis in children: A retrospective study. J Pediatr Orthop. 1983;2(3):249-54.

17. Grey AC, Davies AM, Mangham DC, Grimer RJ, Ritchie DA. The 'penumbra sign' on T1-weighted MR imaging in subacute osteomyelitis: Frequency, cause and significance. Clin Radiol.1998;53(8):587-92.

18. Olasinde AA, Oluwadiya KSAO. Treatment of Brodie's abscess: excellent results from curettage, bone grafting and antibiotics. Singapore Med J. 2011;52(6):436. 